\title{
Bose-Einstein Condensation Temperature of Homogenous Weakly Interacting Bose Gas in Variational Perturbation Theory Through Six Loops
}

\author{
Boris Kastening \\ Institut für Theoretische Physik, Freie Universität Berlin, Arnimallee 14, D-14195 Berlin, Germany \\ email: boris.kastening@physik.fu-berlin.de
}

(Dated: March 2003)

\begin{abstract}
We compute the shift of the transition temperature for a homogenous weakly interacting Bose gas in leading order in the scattering length $a$ for given particle density $n$. Using variational perturbation theory through six loops in a classical three-dimensional scalar field theory, we obtain $\Delta T_{c} / T_{c}=$ $1.25 \pm 0.13 a \mathrm{n}^{1 / 3}$, in agreement with recent Monte-Carlo results.

PACS numbers: 03.75.Hh, 05.30.Jp, 12.38.Cy
\end{abstract}

A dilute homogenous Bose gas with particle density $n$, where the scattering length $a$ is small compared to the interparticle spacing $\sim n^{-1 / 3}$ is a fine example of how, under the right conditions, collective effects can generate strongly coupled modes from microscopic degrees of freedom exhibiting non-zero, but arbitrarily weak interactions. These conditions are met when the temperature is close to the transition temperature for Bose-Einstein condensation (BEC). Naive perturbation theory (PT) then breaks down for physical quantities sensitive to the collective long-wavelength modes. One of the basic questions is about the nature and size of the shift of the BEC transition temperature due to the interactions. Although attempts at the problem have a long history [1, 2], only the recent advent of experimental realizations of BEC in dilute gases have prompted considerable work to finally solve the problem both qualitatively and quantitatively [3, 4, 5, 6, 17, 8, 9, 10, 11, 12, 13, 14, 15, 16, 17, 18, 19, 20]. Here we treat the case of a homogenous gas as opposed to, e.g., the case of a harmonic trap [21].

The appropriate formal framework for the treatment of the many-particle Schrödinger equation describing a gas of identical spin- 0 bosons is non-relativistic $(3+1)$ dimensional field theory. Being interested only in equilibrium quantities, we switch to imaginary time $\tau=i t$ and consider the Euclidean action

$S_{3+1}=\int_{0}^{\beta} d \tau \int d^{3} x\left[\psi^{*}\left(\frac{\partial}{\partial \tau}-\frac{1}{2 m} \nabla^{2}-\mu\right) \psi+\frac{2 \pi a}{m}\left(\psi^{*} \psi\right)^{2}\right]$

where $m$ is the mass of the bosons and $\mu$ the chemical potential and we work in units where $k_{B}=\hbar=1$. For the description of the physics involving the long-wavelength modes, the modes with non-zero Matsubara frequencies may be integrated out from the theory, leaving a threedimensional classical field theory. This is, in general, a complicated procedure, but, as a first approximation, we may simply ignore the dependence of $\psi$ on $\tau$ and obtain the three-dimensional effective Euclidean action

$$
S_{3}=\int d^{3} x\left[\frac{1}{2}|\nabla \phi|^{2}+\frac{r_{\text {bare }}}{2} \phi^{2}+\frac{u}{24}\left(\phi^{2}\right)^{2}\right]
$$

with the identifications $\psi=\sqrt{m T}\left(\phi_{1}+i \phi_{2}\right), r_{\text {bare }}=$ $-2 m \mu, u=48 \pi a m T$. For details on the matching beyond this approximation and why, for our purposes, it is indeed justified to use (2), see Ref. 15]. It is convenient to generalize the model (2) to an $\mathrm{O}(N)$ field theory, where $\phi=\left(\phi_{1}, \ldots, \phi_{N}\right), \phi^{2} \equiv \phi_{a} \phi_{a}$. This allows to make contact with the exactly known large- $N$ result for $\Delta T_{c}$ [8].

It has been argued convincingly 7, 8, 14 that the shift of the transition temperature away from the noninteracting value

$$
T_{0}=\frac{2 \pi}{m}\left[\frac{n}{\zeta(3 / 2)}\right]^{2 / 3}
$$

may be expanded as

$$
\frac{\Delta T_{c}}{T_{0}}=c_{1} a n^{1 / 3}+\left[c_{2}^{\prime} \ln \left(a n^{1 / 3}\right)+c_{2}^{\prime \prime}\right]\left(a n^{1 / 3}\right)^{2}+\cdots
$$

and that the constant $c_{1}$ is independent of the non-zero Matsubara modes and can be computed within the above three-dimensional field theory (2) (for the exact value of $c_{2}^{\prime}$ and an approximate evaluation of $c_{2}^{\prime \prime}$, see Ref. [15]).

Apart from the fact that the leading dependence of $\Delta T_{c}$ on $a$ has only been resolved recently [7], attempts at the determination of $c_{1}$ have produced results from -0.93 9] to 4.7 [2] as has been summarized for instance in Ref. [13], see also Fig. 2 below. The various approaches have different weaknesses and we comment on some of them below. It appears that the most reliable results so far are obtained by Monte-Carlo simulations (MC) [12, 13].

Improving perturbation theory by a variational principle is an old idea 22]. Earlier attempts using resummed PT to determine $\Delta T_{c}[11,17,18,19]$ have employed the linear $\delta$ expansion (LDE). These were criticized in Refs. 20, 23], where Kleinert's field theoretic variational perturbation theory (VPT, see [26, 27, 28] and Chapters 5 and 19 of the textbooks [29] and 30], respectively) was used through five loops. We extend this calculation to six loops with a different treatment of the one-loop term, which is absent here. Our first non-zero coefficient arises only at three loops. In consequence, we obtain a $30 \%$ larger result than Ref. [20]. We comment on the issue at the end of the paragraph following Eq. (8) below.

After the interaction is turned on, we can either tune the temperature by $\Delta T_{c}$ or the particle density by $\Delta n$ to bring the system back to the transition, keeping the respective other quantity fixed. Eq. (3) relates the two shifts in leading order [7],

$$
\frac{\Delta T_{c}}{T_{0}}=-\frac{2}{3} \frac{\Delta n}{n} .
$$


In other words, we may determine $c_{1}$ by computing $\Delta n$ at the critical point. $\Delta n$ is related to the interactioninduced shift of $\left\langle\phi^{2}\right\rangle$ in the three-dimensional theory by

$$
\Delta n=\Delta\left\langle\psi^{*} \psi\right\rangle=m T \Delta\left\langle\phi^{2}\right\rangle .
$$

Combining (3), (4), (5) and (6), $c_{1}$ is given by

$$
c_{1}=-\left.\frac{256 \pi^{3}}{[\zeta(3 / 2)]^{4 / 3}} \frac{\Delta\left\langle\phi^{2}\right\rangle}{N u}\right|_{\text {crit. }} \approx-\left.2206.19 \frac{\Delta\left\langle\phi^{2}\right\rangle}{N u}\right|_{\text {crit. }}
$$

and the remaining task is to compute the critical limit of $\Delta\left\langle\phi^{2}\right\rangle / N u$ in the three-dimensional theory. We have

$$
\Delta\left\langle\phi^{2}\right\rangle=N \int \frac{d^{3} p}{(2 \pi)^{3}}\left[G(p)-G_{0}(p)\right],
$$

where $G$ and $G_{0}$ are the interacting and the free propagator, respectively. The theory (2) is super-renormalizable and only $r_{\text {bare }}$ has to be tuned to cancel UV divergences (a further divergence in the free energy is of no interest to us here). A convenient renormalization scheme defines the renormalized quantity $r=r_{\text {bare }}-\Sigma(0)$, where $\Sigma(p)$ is the self-energy. The full propagator reads then $G(p)=1 /\left\{p^{2}+r-[\Sigma(p)-\Sigma(0)]\right\}$ and $r=0$ corresponds to the critical point, where BEC takes place. Consequently, $G_{0}(p)=1 /\left(p^{2}+r\right) 19$. Another popular scheme is to use $G_{0}(p)=1 / p^{2}$ [1, 17, 18, 20, 23]. This generates an unnatural one-loop contribution to $\Delta T_{c}$ even in the absence of interactions. Although this contribution vanishes as $r \rightarrow 0$, it strongly influences resummation and is responsible for the small value of $c_{1}$ in Ref. 20].

Perturbation theory organizes $\Delta\left\langle\phi^{2}\right\rangle / N u$ in a loop expansion

$$
\frac{\Delta\left\langle\phi^{2}\right\rangle}{N u}=\sum_{l=1}^{\infty} a_{l} u_{r}^{l-2}, \quad u_{r} \equiv \frac{N u}{4 \pi r^{1 / 2}} .
$$

At one loop, there is no interaction and therefore the difference in (8) vanishes. There is also no two-loop contribution since our renormalization scheme subtracts all graphs containing momentum-independent self-energy insertions and there is no two-loop graph without such an insertion. This reflects the fact that the one-loop correction of the propagator merely renormalizes the chemical potential, which does not lead to a shift in the critical temperature [7, 16]. The three-loop contribution is

$$
a_{3} u_{r}=\frac{1}{N u}\left[\bigcirc-\frac{\left(1+\frac{2}{N}\right) \ln \frac{4}{3}}{576 \pi^{2}} u_{r}\right.
$$

where the dashed box means that the enclosed self-energy contribution has to be taken at zero external momentum. The three-loop case exhibits the only type of divergence appearing at any loop order of the calculation, the "sunset" subdivergence which causes the two diagrams in (10) to be UV divergent. One may either regulate each diagram and remove the regulator from the finite difference or, much simpler, perform the momentum integrations only after the subtraction to arrive at the given result.
To save space, it is convenient to define an operator $\mathcal{R}$ that recursively subtracts the zero-momentum contributions of all self-energy insertions of a given diagram. E.g., the combination of diagrams in Eq. (10) is then represented by $\mathcal{R}$ applied to the first diagram. The diagrams through six loops, their weights and $\mathrm{O}(N)$ symmetry factors as well as the numerical values of the corresponding integrals are given in Table प The latter were obtained by own computations and confirmed by extracting them from Ref. 24]. Details will be presented elsewhere [25]. The values for the diagrams through five loops are consistent with those in Ref. 19] [we have more precise results for diagrams 5-4 and 5-5, though; the five-loop results of 17. have to be taken with care since at least two of the diagrams (5-2 and 5-3) are wrong there, while the corresponding coefficient $a_{5}$ is off by $\left.10 \%\right]$.

The resulting perturbative coefficients are $a_{1}=a_{2}=$ $0, a_{3}=-5.06047 \times 10^{-5}, a_{4}=2.99626 \times 10^{-6}, a_{5}=$ $-1.93583 \times 10^{-7}, a_{6}=1.31373 \times 10^{-8}$ for $N \rightarrow \infty$ and $a_{1}=a_{2}=0, a_{3}=-1.01209 \times 10^{-4}, a_{4}=2.99626 \times 10^{-5}$, $a_{5}=-1.19872 \times 10^{-5}, a_{6}=5.85519 \times 10^{-6}$ for $N=2$.

To extract the $r \rightarrow 0$ limit of (9), we need to apply a resummation algorithm. The alternating signs of the coefficients $a_{l}$ suggest to use a Borel-type method, but since we have no information on the distance of the first singularity of the Borel sum from the origin in the complex plane, we proceed differently and use VPT. This method has proven to yield accurate results for critical exponents [27, 28, 30] and amplitude ratios [31] in the present context of critical phenomena. The crucial attractive feature of VPT for our purpose is that it allows to implement the correct Wegner exponent of approach to scaling $\omega\left[=\beta^{\prime}\left(g^{*}\right)\right.$ in a renormalization group treatment] [32]. For quantities like $\Delta\left\langle\phi^{2}\right\rangle / N u$ that remain finite at the critical point, this approach has, in our renormalization scheme, an expansion

$$
\frac{\Delta\left\langle\phi^{2}\right\rangle}{N u}=\sum_{m=0}^{\infty} f_{m} u_{r}^{-m \omega^{\prime}},
$$

where $\omega^{\prime}=2 \omega /(2-\eta)$ and $\eta$ is the anomalous dimension of the critical propagator.

For a truncated partial sum $\sum_{l=1}^{L} a_{l} u_{r}^{l-2}$ of (9), Kleinert's VPT requires replacing

$$
u_{r}^{l-2} \rightarrow(t \hat{u})^{l-2}\left\{1+t\left[\left(\frac{\hat{u}}{u_{r}}\right)^{\omega^{\prime}}-1\right]\right\}^{-(l-2) / \omega^{\prime}}
$$

(note that this is an identity for $t=1$ ), reexpanding the resulting expression in $t$ through $t^{L-2}$, setting $t=1$ and then optimizing in $\hat{u}$, where optimizing is done in accordance with the principle of minimal sensitivity [33] and in practice means finding appropriate stationary or turning points. That is, we replace

$$
u_{r}^{l-2} \rightarrow \hat{u}^{l-2} \sum_{k=0}^{L-l}\left(\begin{array}{c}
-(l-2) / \omega^{\prime} \\
k
\end{array}\right)\left[\left(\frac{\hat{u}}{u_{r}}\right)^{\omega^{\prime}}-1\right]^{k}
$$

and optimize the resulting expression in $\hat{u}$. For $u_{r} \rightarrow \infty$, we obtain the $L$-loop approximation of the $r \rightarrow 0$ limit 


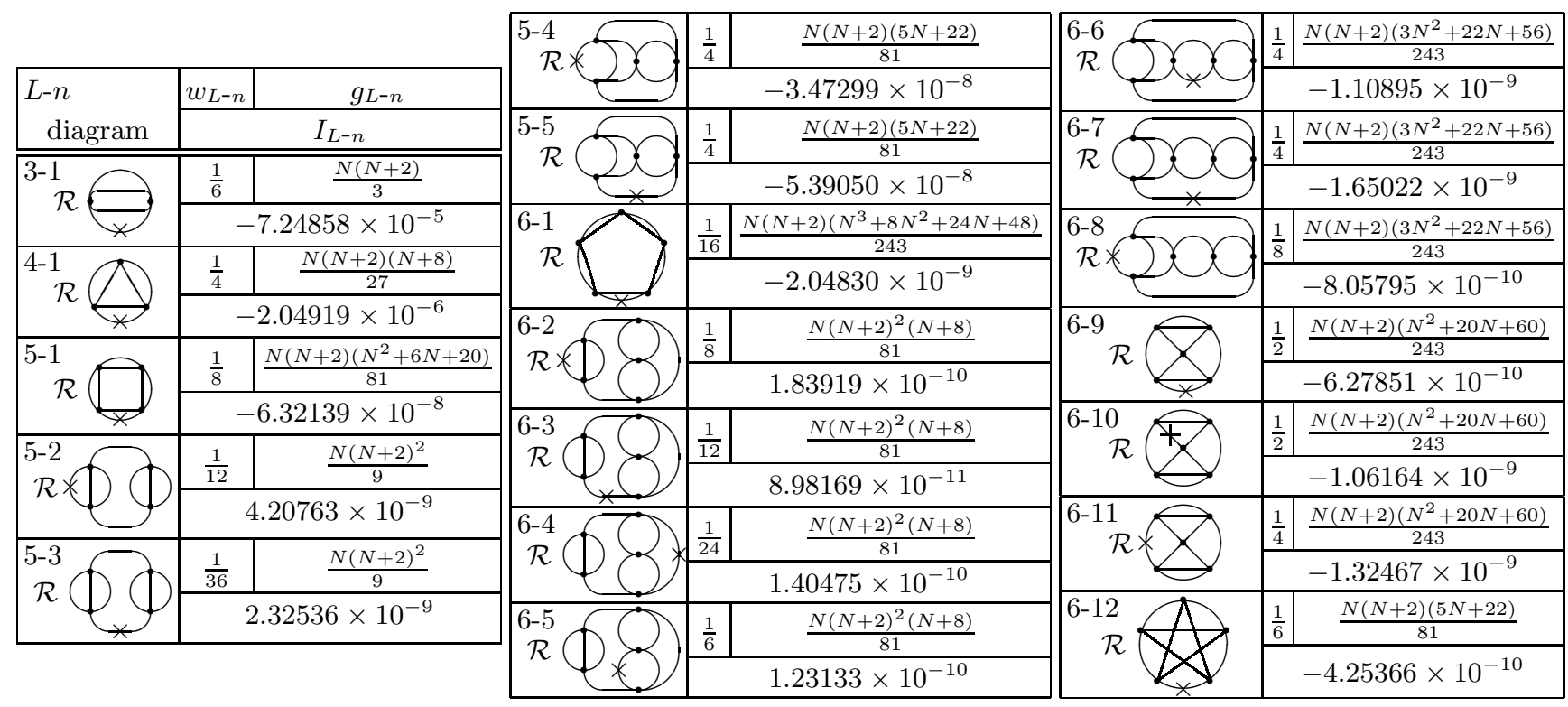

TABLE I: Diagrams from three through six loops, their weights $w_{L-n}, O(N)$ symmetry factors $g_{L-n}$ and numerical results $I_{L-n}$ for the corresponding integrals for $r=1$. The contribution of each diagram to $\left\langle\phi^{2}\right\rangle$ is $(-u)^{L-1} r^{1-L / 2} w_{L-n} g_{L-n} I_{L-n}$.

of $\Delta\left\langle\phi^{2}\right\rangle / N u$

$$
f_{0}^{(L)}=\operatorname{opt}_{\hat{u}}\left[\sum_{l=1}^{L} a_{l} \hat{u}^{l-2} \sum_{k=0}^{L-l}\left(\begin{array}{c}
-(l-2) / \omega^{\prime} \\
k
\end{array}\right)(-1)^{k}\right] .
$$

Results are only available starting at four loops, since two non-zero perturbative coefficients are necessary for VPT to work.

We first treat the $N \rightarrow \infty$ limit, where $\eta=0$ and $\omega=\omega^{\prime}=1$ (see, e.g., Ref. [30]). For large $N$, the only surviving diagrams are 3-1, 4-1, 5-1, 6-1 and their generalization to higher orders, which are all easy to compute. The corresponding resummed results start out as $f_{0}^{(4)}=-8.54677 \times 10^{-4}, f_{0}^{(5)}=-9.14163 \times 10^{-4}$, $f_{0}^{(6)}=-9.55894 \times 10^{-4}$. We have plotted $f_{0}^{(4)}, \ldots, f_{0}^{(40)}$ in Fig. 11. They are seen to converge, albeit slowly, to the exact result $\Delta\left\langle\phi^{2}\right\rangle / N u=-1 / 96 \pi^{2}=-1.05543 \times 10^{-3}$ [8]. We will return to the issue of slow convergence for the $N \rightarrow \infty$ limit below.

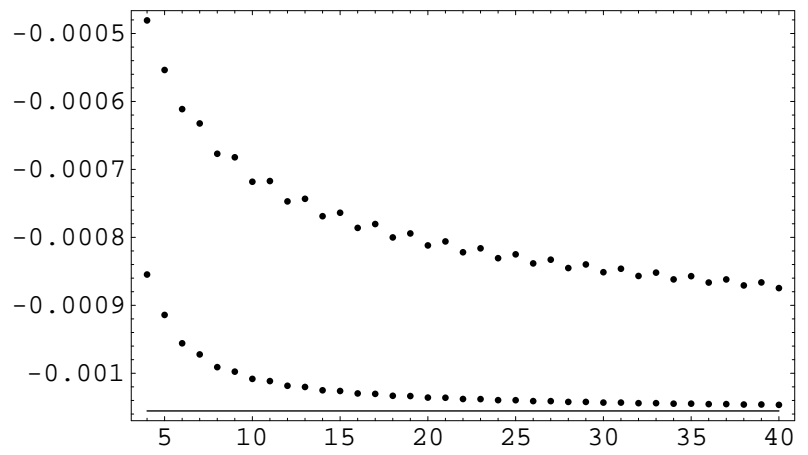

FIG. 1: Resummed results for $\Delta\left\langle\phi^{2}\right\rangle / N u$ as a function of the number of loops $L$ for $N \rightarrow \infty$. Upper dots: LDE. Lower dots: VPT. Solid line: exact result.

The LDE used in Refs. 11, 17, 18, 19] corresponds to arbitrarily setting $\omega^{\prime}=2$. The corresponding values start out as $f_{0}^{(4)}=-4.80756 \times 10^{-4}, f_{0}^{(5)}=-5.53705 \times 10^{-4}$, $f_{0}^{(6)}=-6.11288 \times 10^{-4}$. We have included $f_{0}^{(4)}, \ldots, f_{0}^{(40)}$ in Fig. 1 to demonstrate the at best very slow convergence. There are attempts to accelerate it [18], but it has been argued in Ref. 23 that LDE is inapplicable to field theory whenever there is an irrational exponent of approach to scaling.

Now we turn to the case $N=2$. We employ two approaches when determining the $r \rightarrow 0$ limit of $\Delta\left\langle\phi^{2}\right\rangle / N u$. One of them uses the known values $\omega=0.79 \pm 0.01$, $\eta=0.037 \pm 0.003$ (see, e.g., Ref. [30]) and thus $\omega^{\prime}=$ $0.805 \pm 0.011$ for $N=2$, and the other self-consistently determines $\omega^{\prime}$ at each order. With $\omega^{\prime}=0.805$ (its uncertainty \pm 0.011 causes a $1 \%$ uncertainty of the fixed $-\omega^{\prime}$ six-loop estimate for $c_{1}$ ) we obtain $f_{0}^{(4)}=-4.297 \times 10^{-4}$, $f_{0}^{(5)}=-4.814 \times 10^{-4}, f_{0}^{(6)}=-5.103 \times 10^{-4}$, whose translation to $c_{1}$ via (7) is plotted in Fig. 2.

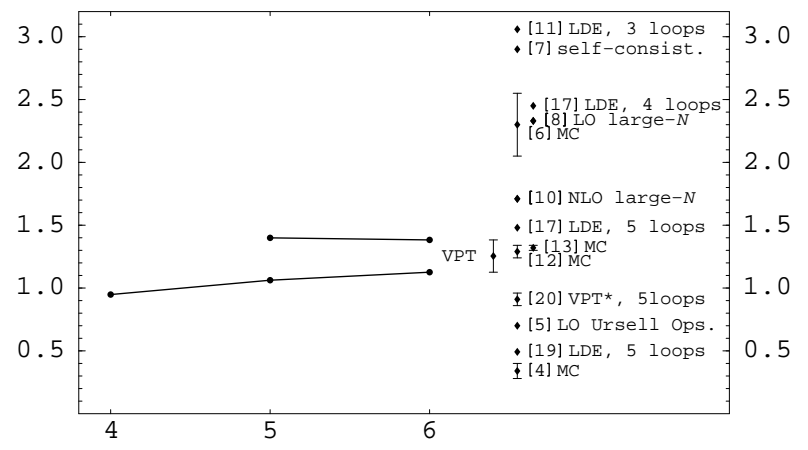

FIG. 2: $\quad c_{1}$ as a function of the number of loops $L$ for $N=2$. Upper dots: self-consistent $\omega^{\prime}$. Lower dots: $\omega^{\prime}=0.805$. The label VPT' indicates the inclusion of a non-zero one-loop term in Ref. 20], which is absent in the present treatment, labeled by VPT. Also included are most results from other sources. 
The expected large- $L$ behavior in VPT has the form $f_{0}+A \exp \left[-B(L-3)^{1-\omega^{\prime}}\right]$ [27, 29, 30]. However, even and odd loop orders in VPT are, in our case, obtained from extrema and turning points, respectively, which causes them to behave slightly differently. This prohibits a trustworthy extrapolation to $L \rightarrow \infty$ at the current loop order. Note that in the large- $N$ limit, where $\omega^{\prime}=1$, the expected large- $L$ behavior turns into $f_{0}+A^{\prime} /(L-3)^{B^{\prime}}$ and an analysis of the data shown in Fig. 1 but extended through $f_{0}^{(150)}$, yields $A^{\prime} \approx 3.4 \times 10^{-4}, B^{\prime} \approx 1.0$.

For determining $\omega^{\prime}$ self-consistently [27, 30], we apply VPT to the quantity $d \ln \left(\Delta\left\langle\phi^{2}\right\rangle / N u\right) / d \ln u_{r}$ and exploit the fact that it vanishes as $u_{r} \rightarrow \infty$ and that its large- $u_{r}$ expansion has the same $\omega^{\prime}$-exponent as $\Delta\left\langle\phi^{2}\right\rangle / N u$. That is, at each loop order we tune $\omega^{\prime}$ such that the optimization procedure leads to a vanishing approximation for the large- $u_{r}$ limit of $d \ln \left(\Delta\left\langle\phi^{2}\right\rangle / N u\right) / d \ln u_{r}$. This $\omega^{\prime}$ is then used at the same loop order in (14). Our results are $f_{0}^{(5)}=-6.343 \times 10^{-4}$ and $f_{0}^{(6)}=-6.268 \times 10^{-4}$, with corresponding $\omega^{\prime}$-values $\omega^{\prime(5)}=0.6212$ and $\omega^{\prime(6)}=0.6381$.
Via (17) they are converted to the estimates for $c_{1}$ plotted in Fig. 2

Taking the average and the difference of our two estimates for $f_{0}^{(6)}$ as the mean value and the total error bar of our final result, respectively, we arrive at $\Delta\left\langle\phi^{2}\right\rangle / N u=$ $-(5.69 \pm 0.58) \times 10^{-4}$ and via (7) at $c_{1}=1.25 \pm 0.13$. This is in agreement with the recent $\mathrm{MC}$ data $c_{1}=1.32 \pm 0.02$ [13] and $c_{1}=1.29 \pm 0.05[12]$ (see Refs. 13, 14] for explanations of the low MC value in Ref. [4]; see Ref. 13] for a critique of the MC calculation of Ref. [6]). Fig. 2] shows a comparison with most available results.

In summary, we provide a theoretically well-founded resummed perturbative result for $c_{1}$, comparing favorably with recent Monte-Carlo data.

The author is grateful to $\mathrm{H}$. Kleinert for important suggestions, many discussions, and a careful reading of the manuscript; also to E. Braaten for many helpful comments on the manuscript and to B. Nickel and T. Binoth for useful communications.
[1] T.D. Lee and C.N. Yang, Phys. Rev. 112, 1419 (1957). K. Huang, in Studies in Statistical Mechanics, edited by J. de Boer and G.E. Uhlenbeck (North-Holland Publishers, Amsterdam, 1964), Vol. II, p. 1; T. Toyoda, Ann. Phys. (N.Y.) 141, 154 (1982).

[2] H.T.C. Stoof, Phys. Rev. A 45, 8398 (1992).

[3] M. Bijlsma and H.T.C. Stoof, Phys. Rev. A 54, 5085 (1996); K. Huang, Phys. Rev. Lett. 83, 3770 (1999); E.J. Mueller, G. Baym and M. Holzmann, J. Phys. B 34, 4561 (2001).

[4] P. Grüter, D. Ceperley and F. Laloë, Phys. Rev. Lett. 79, 3549 (1997).

[5] M. Holzmann, P. Grüter and F. Laloë, Eur. Phys. J. B 10, 739 (1999).

[6] M. Holzmann and W. Krauth, Phys. Rev. Lett. 83, 2687 (1999).

[7] G. Baym, J.-P. Blaizot, M. Holzmann, F. Laloë and D. Vautherin, Phys. Rev. Lett. 83, 1703 (1999).

[8] G. Baym, J.-P. Blaizot and J. Zinn-Justin, Europhys. Lett. 49, 150 (2000).

[9] M. Wilkens, F. Illuminati, M. Kraemer, J. Phys. B 33, L779 (2000).

[10] P. Arnold and B. Tomášik, Phys. Rev. A 62, 063604 (2000).

[11] F.F. de Souza Cruz, M.B. Pinto and R.O. Ramos, Phys. Rev. B 64, 014515 (2001).

[12] V.A. Kashurnikov, N.V. Prokof'ev and B.V. Svistunov, Phys. Rev. Lett. 87, 120402 (2001); N.V. Prokof'ev and B.V. Svistunov, cond-mat/0103146 1.

[13] P. Arnold and G. Moore, Phys. Rev. Lett. 87, 120401 (2001); Phys. Rev. E 64, 066113 (2001).

[14] M. Holzmann, G. Baym, J.-P. Blaizot and F. Laloë, Phys. Rev. Lett. 87, 120403 (2001).

[15] P. Arnold, G. Moore and B. Tomášik, Phys. Rev. A 65, 013606 (2001).
[16] G. Baym, J.-P. Blaizot, M. Holzmann, F. Laloë and D. Vautherin, Eur. Phys. J. B 24, 104 (2001).

[17] F.F. de Souza Cruz, M.B. Pinto, R.O. Ramos and P. Sena, Phys. Rev. A 65, 053613 (2002).

[18] J.-L. Kneur, M.B. Pinto and R.O. Ramos, Phys. Rev. Lett. 89, 210403 (2002); cond-mat/0207295 2.

[19] E. Braaten and E. Radescu, Phys. Rev. A 66, 063601 (2002); Phys. Rev. Lett. 89, 271602 (2002).

[20] H. Kleinert, cond-mat/0210162 2 .

[21] S. Giorgini, L.P. Pitaevskii and S. Stringari, Phys. Rev. A 54, R4633 (1996); P. Arnold and B. Tomášik, Phys. Rev. A 64, 053609 (2001).

[22] V.I. Yukalov, Moscow Univ. Phys. Bull. 31, 10 (1976).

[23] B. Hamprecht and H. Kleinert, hep-th/0302116 1.

[24] B.G. Nickel, D.I. Meiron and G.A. Baker, Jr., Univ. of Guelph Report, 1977, unpublished; M. Muthukumar and B. Nickel, J. Chem. Phys. 80, 5839 (1984).

[25] B. Kastening, in preparation.

[26] H. Kleinert, Phys. Lett. A 207, 133 (1995).

[27] H. Kleinert, Phys. Rev. D 57, 2264 (1998); Phys. Rev. D 58, 107702 (1998).

[28] H. Kleinert, Phys. Rev. D 60, 085001 (1999); Phys. Lett. A 277, 205 (2000).

[29] H. Kleinert, Path Integrals in Quantum Mechanics, Statistics and Polymer Physics, 3rd ed. (World Scientific, Singapore, 2003).

[30] H. Kleinert and V. Schulte-Frohlinde, Critical Properties of $\phi^{4}$-Theories, 1st ed. (World Scientific, Singapore, 2001).

[31] H. Kleinert and B. Van den Bossche, Phys. Rev. E 63, 056113 (2001).

[32] F. Wegner, Phys. Rev. B 5, 4529 (1972).

[33] P.M. Stevenson, Phys. Rev. D 23, 2916 (1981). 